\title{
AVALIAÇÃO DA EFETIVIDADE DE MANEJO DAS UNIDADES DE CONSERVAÇÃO DE PROTEÇÃO INTEGRAL EM MINAS GERAIS ${ }^{1}$
}

\author{
Gumercindo Souza Lima², Guido Assunção Ribeiro² e Wantuelfer Gonçalves²
}

\begin{abstract}
RESUMO - Este trabalho buscou avaliar a efetividade de manejo a partir da coleta de dados em campo, através da aplicação de questionários aos gerentes dessas áreas e consulta a dados secundários das instituições gestoras. A metodologia de questionário utilizada foi a "Matriz de Cenários", em que se estabeleceram cenários possíveis, desde a pior até a melhor situação esperada de cada um dos indicadores de manejo escolhidos. Os resultados apontaram que a criação de unidades de conservação, em Minas Gerais, tem ocorrido sem a perspectiva de que estas venham cumprir seus objetivos estabelecidos em sua criação ou definidos na escolha da sua categoria. Constatou-se que apenas uma unidade de conservação apresentava nível satisfatório de manejo e $60 \%$ das unidades exibiram nível insatisfatório de manejo. As unidades nacionais presentes em Minas Gerais apresentavam, em média, resultados melhores que as estaduais, ressaltando-se que, do total, 87\% (34 unidades) não possuíam Plano de Manejo, nem se encontravam em fase de planejamento. O quadro demonstrado neste estudo deixa clara a necessidade de repensar o processo de criação e gestão de unidades de conservação em Minas Gerais. E, em comparação com outros estudos, observou-se que essa é também uma realidade de outros estados, como São Paulo, Rio de Janeiro e Mato Grosso.
\end{abstract}

Palavras-chave: Unidades de conservação, efetividade de manejo e áreas protegidas.

\section{MANAGEMENT EFFECTIVENESS OF CONSERVATION UNITS WITH INTEGRAL PROTECTION IN MINAS GERAIS}

\begin{abstract}
The objective of this work was to evaluate the management effectiveness from data collected in the field, through the application of questionnaires to the managers of those areas and consultation with secondary data of manager institutions. The questionnaire methodology used was the Scenery Matrix, where possible sceneries were established, from the worst situation to the best expected situation for each management indicator chosen. The results showed that the creation of conservation units in the State of Minas Gerais occurs without the expectation that they will fulfill the objectives established in their creation or defined in the choice of their category. Only one conservation unit presented a satisfactory level of management, and $60 \%$ of the units have poor management level. The national units located in the State show, in average, better results than the state ones, and 87\% (34 units) out of the total do not have a Management Plan, and neither of them has a planning process. The picture shown in this study reveals a clear need of thinking over the creation process and management of conservation units in Minas Gerais, and when comparing with other studies, it was observed that unfortunately this is also true in other States such as São Paulo, Rio de Janeiro and Mato Grosso.
\end{abstract}

Keywords: Conservation units, management effectiveness and protected areas.

\footnotetext{
${ }^{1}$ Recebido em 22.10.2004 e aceito para publicação em 20.04.2005.

${ }^{2}$ Departamento de Engenharia Florestal da UFV. E-mail: <gslima@ufv.br>.
} 


\section{INTRODUÇÃO}

O Ministério de Meio Ambiente divulgou recentemente uma nova lista das espécies ameaçadas de extinção, constatando-se que o número de espécies que se encontram nessa situação de risco quase triplicou desde o último levantamento, indicando que a situação de conservação da biodiversidade se encontra deficiente.

Um dos fatores que têm contribuído para esse quadro é o estado de degradação dos ecossistemas, promovido pela expansão agrícola e pelo manejo inadequado dessas áreas. Outro fator é a falta de efetividade do manejo e de proteção ambiental das unidades de conservação no país.

Visando eliminar os riscos da criação desses "parques de papel" foi aprovado, pelo Congresso Nacional, um novo sistema de áreas protegidas ou Sistema Nacional de Unidades de Conservação (SNUC - LEI 9.985, de 18 de julho de 2000), após oito anos de tramitação na Casa Legislativa. O novo sistema vem consolidar uma série de normas e discussão acerca das Unidades de Conservação, suas diversas categorias e sua forma de implantação e manejo.

Segundo Terborgh e Schaik (2002), uma grande fração das unidades de conservação no mundo representa os chamados "parques de papel". Esse termo se refere às unidades de conservação que não foram realmente implantadas e têm apenas uma existência virtual, como linhas desenhadas em mapas oficiais. Cerca de $70 \%$ dos parques tropicais possuem pessoas vivendo em seu interior, segundo Schaik e Rijksen (2002).

Brockelman e Griffiths (2002) apontaram a deficiência na fiscalização como um dos grandes problemas no cumprimento dos objetivos das unidades de conservação.

Criar unidades de conservação sem a perspectiva de implantá-las provocam inúmeros problemas regionais, além prejudicar a relação da instituição e da unidade com as comunidades locais. Dentre esses problemas, podem-se citar: expectativas não-atendidas, desapropriação sem indenização, lucro e produção cessante, desestabilização cultural, déficit tributário pela redução de produção e paralisação do processo de desenvolvimento regional, todas culminando com a insatisfação regional e desgaste do nome e imagem institucionais.
O Estado de Minas Gerais possui 183 unidades de conservação (Figura 1), o equivalente a uma superfície de 2.096.648 ha, ou 3,56\% do território mineiro. As unidades de conservação destinadas à proteção integral, ou seja, nas quais é proibida a coleta ou consumo direto dos recursos, cobrem $0,95 \%$ do território estadual ou 560.695 ha, distribuídos em diversas categorias de manejo, sendo 8 estações ecológicas (8.311 ha), 12 reservas biológicas ( 17.430 ha) e 55 parques (483.634 ha) (CAMARGOS, 2001b).

Os principais objetivos deste trabalho foram:

a)Discutir o processo de criação e implantação de unidades de conservação no Brasil, tendo como estudo de caso o Estado de Minas Gerais.

b) Avaliar a situação das unidades de conservação de proteção integral em Minas Gerais.

c)Avaliar a efetividade de manejo das unidades de conservação de proteção integral em Minas Gerais.

\section{MATERIAL E MÉTODOS}

A avaliação do processo de criação, implantação e manejo de unidades de conservação no Brasil foi feita de forma descritiva, analisando-se os exemplos históricos e elaborando-se uma discussão teórica sobre o processo de criação e de manejo de unidades de conservação.

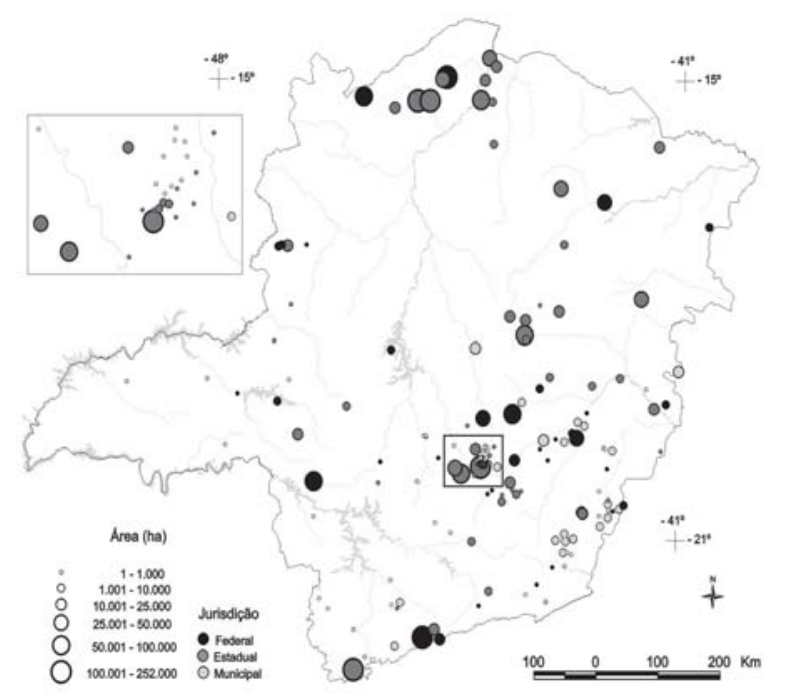

Figura 1 - Distribuição das unidades de conservação em Minas Gerais.

Figure 1 - Distribution of conservation units in Minas Gerais. 
Quanto à avaliação da efetividade do manejo de unidades de conservação, foi desenvolvido um estudo de caso com as unidades de proteção integral do Estado de Minas Gerais.

A metodologia utilizada para avaliar a efetividade do manejo dessas unidades foi uma adaptação da que foi desenvolvida por Faria (1995) para avaliação das unidades de conservação do Estado de São Paulo e a metodologia utilizada por Lemos de Sá (2000), para avaliação das unidades de proteção integral brasileiras.

Foi elaborado um questionário para aplicação aos gerentes das unidades de conservação, visando identificar a situação de cada unidade. Nesse questionário foram construídas várias situações, em que a unidade pode ser encontrada para diversos indicadores de manejo; essa metodologia foi chamada de "matriz de cenários".

A metodologia matriz de cenários proposta para este trabalho foi baseada em trabalho de Faria (1997) e compõe-se de cinco passos seqüenciais, que conduzem a determinação da efetividade do manejo, quais sejam: compilação de dados secundários e estabelecimento de um marco de políticas para o manejo das unidades de conservação; seleção de indicadores e estabelecimento de critérios de avaliação; construção de cenários ótimo e atual; avaliação de indicadores e síntese dos dados; e determinação da efetividade do manejo, como descrito a seguir:

a) Marco de políticas: visa estabelecer um marco de políticas ideais para o manejo das unidades de conservação.

b) Seleção de indicadores: objetiva estabelecer os indicadores que serão utilizados no processo de avaliação do manejo. Os indicadores devem guardar relação direta com os objetivos de manejo das unidades de conservação. Alguns aspectos importantes do manejo são de difícil mensuração, dada a sua grande subjetividade.

c) Construção dos cenários: cenários são instrumentos utilizados comumente no planejamento estratégico, resultantes da articulação seletiva de opções que funcionam como um modelo dinâmico da realidade, tendo como objetivo sondar o futuro e prever os resultados possíveis de alcançar.

d) Avaliação dos indicadores: nesta etapa, os indicadores foram qualificados, tomando-se por base os cenários delineados, utilizando uma escala. O maior valor corresponde a melhor situação, o cenário ótimo; e o menor valor corresponde à pior situação possível, aquela que conflita totalmente com o manejo efetivo da unidade.

e) Determinação da efetividade do manejo: foi estabelecida uma escala (Quadro 1) para qualificação da efetividade do manejo, sendo o resultado obtido pelas comparações dessa escala com o determinante de sua efetividade (Quadro 2).

As respostas aos questionários da matriz de cenários foram dadas pelos chefes das unidades de conservação, entretanto diversas unidades de conservação não possuem gerentes, nem tampouco funcionários, e, quando isso ocorreu, as respostas foram dadas pelos responsáveis indicados pela instituição gestora.

Deve-se entender por efetividade a capacidade da unidade de conservação em cumprir os objetivos para os quais foi criada.

Utilizou-se a avaliação dos seguintes parâmetros para avaliação da situação das unidades de conservação, quanto a efetividade de manejo, demarcação, desapropriação, infra-estrutura, recursos humanos, recursos financeiros, infra-estrutura, recursos financeiros, parcerias, plano de manejo, relação com o entorno, programas de proteção e fiscalização, programas de proteção, controle de incêndios, plano de manejo e pesquisa.

Quadro 1 - Critérios para definição dos graus de efetividade de manejo

Table 1-Criteria for defining degrees of management effectiveness

\begin{tabular}{cc}
\hline Resposta & $\begin{array}{c}\text { Pontuação Obtida em cada Resposta } \\
\text { para Análise do Grau de Efetividade }\end{array}$ \\
\hline 1 & 0 \\
2 & 2,5 \\
3 & 5,0 \\
4 & 7,5 \\
5 & 10,0 \\
\hline
\end{tabular}

Quadro 2 - Critérios para análise dos graus de efetividade de manejo

Table 2 - Criteria for analyzing degrees of management effectiveness

\begin{tabular}{cc}
\hline Pontuação obtida & $\begin{array}{c}\text { Resultado por Característica da } \\
\text { Efetividade de Manejo }\end{array}$ \\
\hline$<2,5$ & Efetividade insatisfatória \\
$2,5-5,0$ & Efetividade pouco satisfatória \\
$5,1-7,5$ & Efetividade medianamente satisfatória \\
$7,6-10,0$ & Efetividade satisfatória \\
\hline
\end{tabular}




\section{RESULTADOS E DISCUSSÃO}

Para análise dos dados não se estabeleceu peso diferenciado para os critérios, embora se considere que alguns critérios avaliados, como desapropriação de terras, número de funcionários, infra-estrutura, plano de proteção e controle de incêndios e plano de manejo sejam vitais para a existência da Unidade de Conservação.

As 23 unidades de proteção integral, 16 parques e 7 estações e reservas, que apresentaram nível insatisfatório de manejo constituem o que se chama de "Parques de Papel", com a ressalva de que, na maioria dos casos, não possuem nenhum hectare desapropriado ou indenizado (9 unidades), nenhum funcionário (19), nenhuma obra de infra-estrutura (18), não recebem nenhum recurso financeiro para a sua gestão (17), nenhum programa de proteção ou controle de incêndios (15) e não possuem plano de manejo (23).

Das 27 unidades de conservação de proteção integral da categoria parque analisadas, $16(60 \%)$ apresentaram nível insatisfatório de manejo, 10 (37\%) um nível médio e apenas o Parque Estadual do Rio Doce exibiu nível satisfatório de manejo, conforme se vê na Figura 2 e no Quadro 3.

Dentre as 12 unidades de conservação das categorias Estação Ecológica e Reserva Biológica analisadas, 7 (58\%) apresentaram nível insatisfatório de manejo, 4 (33\%) nível pouco satisfatório e apenas a Estação

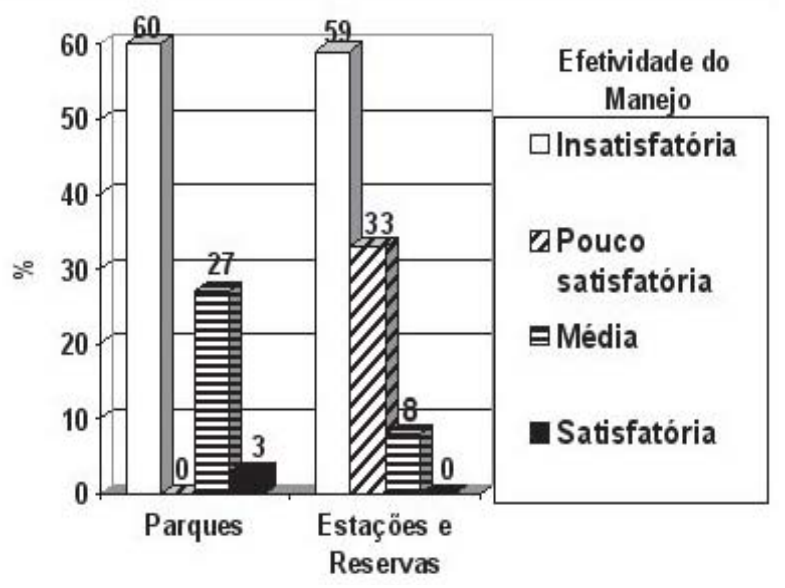

Figura 2 - Efetividade de manejo das unidades de proteção integral em Minas Gerais.

Figure 2-Management effectiveness for completely protected units in Minas Gerais.
Ecológica do Tripuí mostrou nível médio de qualidade de manejo, conforme se vê na Figura 2 e no Quadro 4.

A criação dessas unidades, muitas vezes, acontece para atender a interesses de setores da sociedade ou por obra política dos dirigentes públicos, uma vez que não fazem parte de uma política de planejamento e discussão sobre sua criação e implantação.

Comparando os Parques Nacionais e Estaduais, conclui-se que o setor federal apresenta melhor desempenho e apenas 2 (33\%) Parques Nacionais (P. N. Cavernas do Peruaçu e P. N. da Sempreviva) apresentaram nível insatisfatório de qualidade de manejo, enquanto dos Parques Estaduais, 14 (67\%) foram classificados com do mesmo nível.

Faria (1997), utilizando a metodologia de matriz de cenários, para analisar a situação de oito unidades estaduais de conservação de proteção integral de São Paulo, concluiu que somente o Parque Estadual de Campos do Jordão apresentou nível satisfatório de manejo, três unidades $(37,5 \%)$ exibiram nível de satisfação médio e quatro unidades (50\%), nível pouco satisfatório.

Pires et al. (2000), estudando as unidades de proteção integral do Estado de São Paulo, analisaram a existência ou não de plano de manejo, tamanho da área e bioma representado. Eles chegaram à conclusão de que, das 46 unidades avaliadas, 28 não possuíam plano de manejo e em apenas 1 unidade o plano estava sendo implementado, enquanto 17 se encontravam em fase de elaboração do seu plano. Já, das unidades analisadas em Minas Gerais apenas uma, o Parque Estadual do Rio Doce, possuía plano de manejo elaborado.

Primo e Pellens (2000), analisando 48 unidades de conservação do Estado do Rio de Janeiro quanto à existência de sede administrativa, de centro de visitantes, de plano de manejo e número de funcionários, concluíram que a maioria das unidades possuíam sérios problemas fundiários, além de má distribuição de funcionários nas unidades. Esses autores observaram ainda que apenas $29 \%$ possuíam plano de manejo.

Tocantins e Almeida (2000), analisando as cinco unidades de conservação federais, do Estado do Mato Grosso, concluíram que essas protegem $0,46 \%$ do território do estado e possuem 14 funcionários, ou seja, 1 funcionário para cada 30.000 ha protegidos. Nenhuma das unidades, segundo o estudo, possuía plano de manejo. 


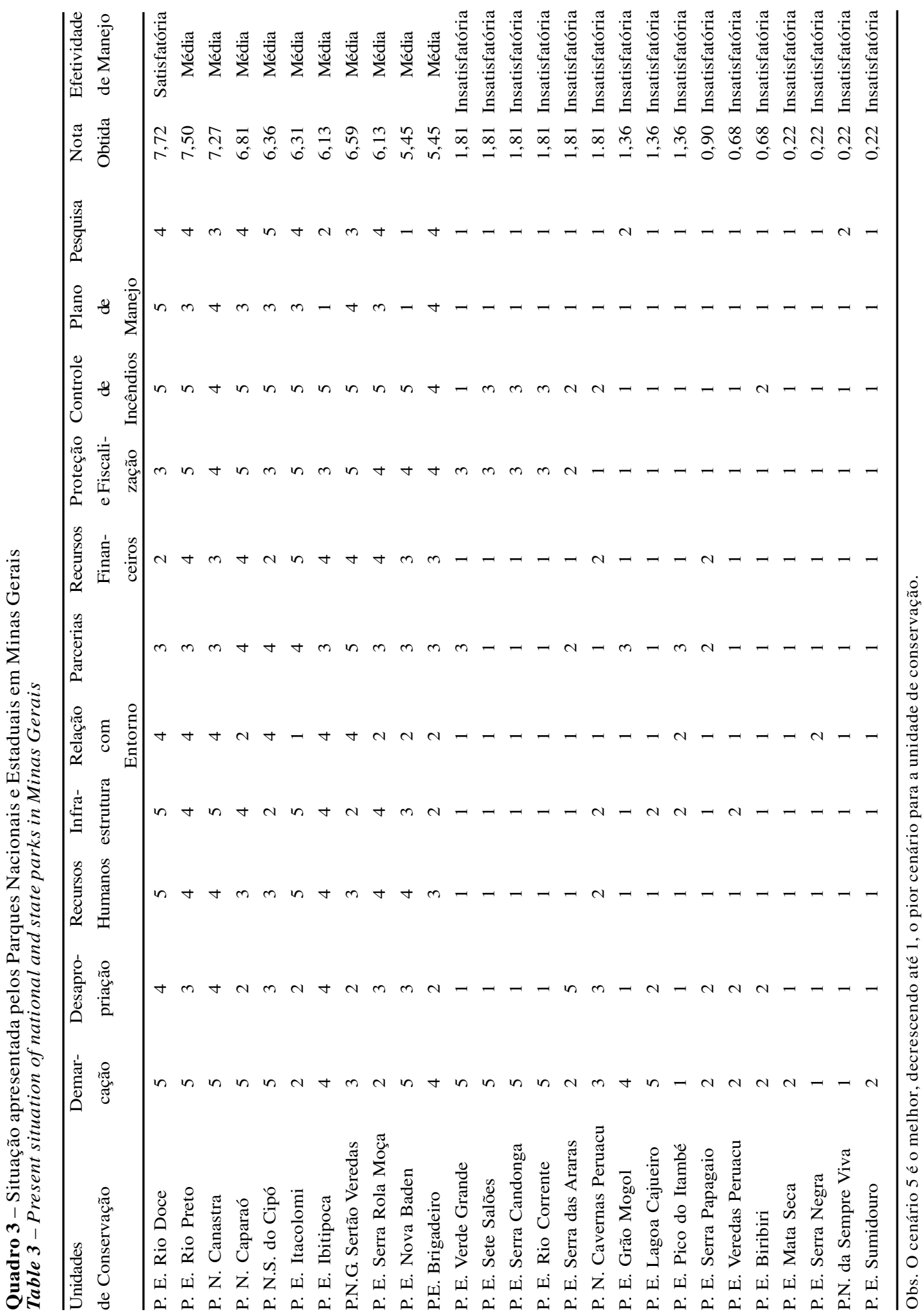

R. Árvore, Viçosa-MG, v.29, n.4, p.647-653, 2005 


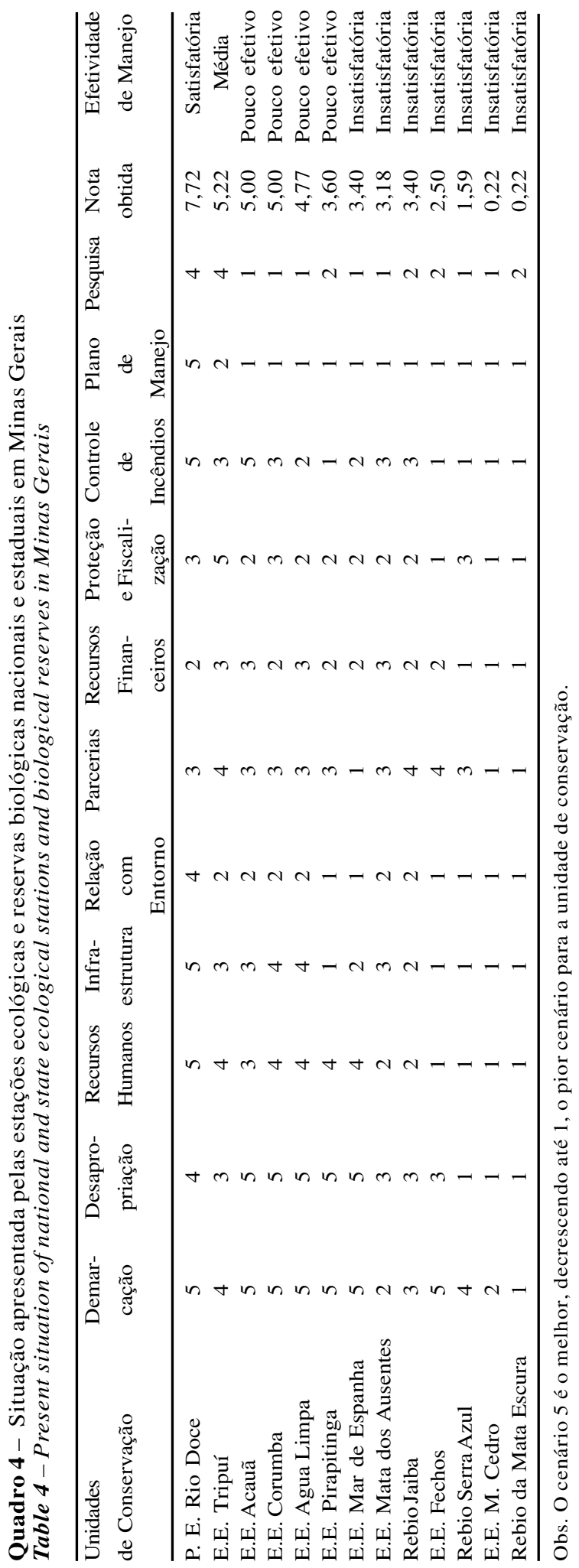

Brito (2000) também analisou as unidades de conservação do Estado do Mato Grosso (14 de proteção integral e 5 de uso sustentável), entre federais e estaduais. Essa autora, analisando o nível de implementação das unidades, concluiu que $63 \%$ possuíam nível inadequado de implementação e $37 \%$, nível regular, e nenhuma unidade dispunha de nível médio ou satisfatório de implementação.

Faria (2002), voltando a analisar três unidades de São Paulo (Parque Estadual da Ilha do Cardoso, Parque Estadual Carlos Botelho e Parque Estadual Morro do Diabo), concluiu que o primeiro possui, atualmente, nível satisfatório de manejo, enquanto as outras duas apresentam nível médio de qualidade de manejo.

O que se percebeu ao longo dos últimos cinco anos, através da análise do processo histórico e dos dados apresentados neste trabalho, é que os dirigentes públicos se preocuparam em criar Unidades de Conservação sem, contudo, planejar sua implantação. Assim, o ato de criação de unidades de conservação no Brasil tornou-se "obra" dos políticos e não mais fruto do planejamento e do anseio da sociedade organizada e das entidades de pesquisa.

\section{CONCLUSÃO}

O presente estudo demonstrou, a partir dos resultados, que a criação de unidades de conservação em Minas Gerais tem ocorrido sem a perspectiva de que estas venham cumprir seus objetivos, e entendese que esta deve ser uma atitude a ser repensada pelo poder público.

Esta pesquisa revelou que 23 unidades $(60 \%)$ podem ser consideradas "parques de papel”, ou seja, apresentavam nível insatisfatório de manejo, mas em apenas uma unidade esse nível era satisfatório. Além disso, 87\% (34 unidades) não possuíam plano de manejo, nem se encontravam em fase de planejamento.

Deve-se, portanto, questionar se é justo usar recursos públicos cada vez mais escassos, promover a desapropriação de moradores locais, provocar a insegurança regional pela posse das terras, apregoar expectativas de ação e de conservação sem nenhuma programação de captação de recursos para gerenciamento da área ou para indenização dos proprietários. $O$ dirigente público poderá ser, à luz do direito e a bem da responsabilidade pública, responsabilizado pelos "Parques de Papel" e pelas expectativas não atendidas. 
O quadro apresentado neste estudo deixa clara a necessidade de repensar os processos de criação e gestão de unidades de conservação em Minas Gerais, e, ao se comparar com outros estudos, observa-se que esta é uma realidade também, em outros estados, como São Paulo, Rio de Janeiro e Mato Grosso.

Urge que se reveja a política ora implantada para gestão das unidades de conservação, ou estará comprometida a proteção da biodiversidade no Brasil.

\section{REFERÊNCIAS BIBLIOGRÁFICAS}

BRITO, M. A. Avaliação do nível de implementação das unidades de conservação do Estado do Mato Grosso. In: CONGRESSO BRASILEIRO DE UNIDADES DE CONSERVAÇÃO, 2., 2000, Campo Grande Anais... Trabalhos técnicos, Campo Grande: 2000. v.2. p. 645-653.

BROCKELMAN,W. Y.;GRIFFITHS, M. Mecanismos de fortalecimento das áreas protegidas. In: Tornando os parques eficientes: estratégias para conservação da natureza nos trópicos. Curitiba: Universidade Federal do Paraná, 2002. p. 290-304.

CAMARGOS, R. Discutindo a gestão participativa de unidades de conservação. Revista Ação Ambiental, v. 4, n. 19, p.14-17, 2001.

CAMARGOS, R. Unidades de conservação em Minas Gerais: levantamento e discussão. Belo Horizonte: Fundação Biodiversitas, 2001. 67p. (Publicações Avulsas, 2).

FARIA, H. H. Procedimento para medir a efetividade do manejo de áreas silvestres protegidas. Revista do Instituto Florestal, v. 7, n. 1, p.35-55, 1995.

FARIA, H. H. Avaliação da efetividade do manejo de unidades de conservação: como proceder? In: CONGRESSO BRASILEIRO DE UNIDADES DE CONSERVAÇÃO, 1997, Curitiba. Anais.... Curitiba: 1997. p. 478-499.
FARIA, H. H. Estado da gestão de três unidades de conservação do Estado de São Paulo. In: CONGRESSO BRASILEIRO DE UNIDADES DE CONSERVAÇÃO, 3., 2002, Fortaleza. Anais... Fortaleza: 2002. p. 289-303.

LEMOS DE SÁ, R. Unidades de conservação: espaços ameaçados ou áreas protegidas. Brasília: 2000. 32 p. (Relatório Técnico).

PIRES, A. M. Z. C. R.; SANTOS, J. E.; PIRES, J. S. Conservação da biodiversidade: análise da situação de unidades de proteção integral do Estado de São Paulo. In: CONGRESSO BRASILEIRO DE UNIDADES DE CONSERVAÇÃO, 2., 2000, Campo Grande. Trabalhos Técnicos, Campo Grande: 2000. v.2. p. 618-627.

PRIMO, P. B. S.; PELLENS, R. A situação atual das unidades do Estado do Rio de Janeiro. In: CONGRESSO BRASILEIRO DE UNIDADES DE CONSERVAÇÃO, 2, 2000, Campo Grande. Trabalhos Técnicos, Campo Grande: 2000. v.2. p. 628-637.

SCHAIK, C. V.; RIJKSEN, H. D. Projetos integrados de conservação e desenvolvimento: problemas e potenciais. In: TORNANDO os parques eficientes: estratégias para conservação da natureza nos trópicos. Curitiba: Universidade Federal Paraná, 2002. p. 37-51.

TERBORGH, J.; SCHAIK, C. V. Por que o mundo necessita de parques? In: Tornando os parques eficientes: estratégias para conservação da natureza nos trópicos. Curitiba: Universidade Federal do Paraná, 2002. p. 25-36.

TOCANTINS, N.; ALMEIDA, A. F. As unidades de conservação federais: uma análise da realidade matogrossense. In: CONGRESSO BRASILEIRO DE UNIDADES DE CONSERVAÇÃO, 2, 2000, Campo Grande. Trabalhos Técnicos, Campo Grande: 2000. v.2. p. 638-644. 